\title{
Statistical Study of Process Parameters Effect on Crystallinity of Electrospun Polyamide 6,6 Fibres
}

\section{Fabiola Navarro Pardo ${ }^{1,2 *}$, Ana Rivera-López ${ }^{2}$, Víctor Sánchez-Labastida ${ }^{3}$, Ana Martínez- Hernández ${ }^{1,2}$, Armando Almendarez-Camarillo ${ }^{4}$ and Carlos Velasco-Santos ${ }^{1,2}$}

1 Instituto Tecnológico de Querétaro, División de Estudios de Posgrado e Investigación, Av. Tecnológico s/n, esq. Gral. Mariano Escobedo, Col. Centro Histórico, C.P. 76000, Santiago de Querétaro, Querétaro, México; E-Mails: almh72@gmail.com (A.L.M.-H.); cylaura@gmail.com (C. V.-S.);

2 Centro de Física Aplicada y Tecnología Avanzada, Universidad Nacional Autónoma de México Campus Juriquilla, Apartado Postal 1-1010, Querétaro, Qro. C.P. 76000 México; E-Mails: anarivera2000@yahoo.com (A. R.-L.);

3 Instituto Tecnológico de Orizaba, Ingeniería Química, Av. Tecnológico No. 852, Col. E. Zapata, C.P. 94320, Orizaba Veracruz, México; E-Mails: vicsanlab@hotmail.com (V. S.-L.);

4 Departamento de Ingeniería Química, Instituto Tecnológico de Celaya, Av. Tecnológico y Antonia García Cubas, s/n Col. Fovissste, C.P. 38010. Celaya, Guanajuato, México; Email: armando@iqcelaya.itc.mx (A.A-C).

* Author to whom correspondence should be addressed; E-Mail: faby.navpard@gmail.com (F.N.-P.); Tel.: +52-442-238-1145; Fax: +52-442-238-1165.

Received: 1 April 2014 / Published: 6 June 2014

Abstract: Scaffolds play an important role in tissue engineering; therefore a variety of
investigations have been conducted using different strategies for obtaining these
architectures. Electrospinning of polyamides has been used for obtaining porous nanofibres
that can be employed in a variety of applications; among them their use for fabricating
tissue scaffolds has attracted a lot of interest. In order to successfully apply these
nanofibres in the biomedical field it is important to investigate the diverse parameters
involved in this process. Fibre diameter, network morphology and mechanical properties
are some of the most important features that can be tailored to mimic specific target tissues.
Furthermore, crystallinity of the polymer is related to the mechanical response and 
biodegradability. This work aims to present a better insight into the structural features of polyamide 6,6 fibres obtained from electrospinning technique by evaluating the crystallinity percentage when processed at different conditions. The parameters studied in this work are the solution concentration, flow rate, voltage applied and distance from the tip of the needle to the collector surface. Differential scanning calorimetry was used to determine the crystallinity of the samples. The morphology of the nanofibres was observed by scanning electron microscopy. The chemical structure of the nanofibres was analysed by infrared and Raman spectroscopies. The results obtained showed those parameters that attained major changes on the crystallinity of the polyamide 6,6 nanofibres.

Keywords: polyamide 6,6; electrospinning parameters; crystallinity, structural changes.

\section{Introduction}

Electrospinning is an efficient and versatile process to obtain fine porous fibres with diameters ranging from several microns to tens of nanometers. Electrospun fibres can be adapted in different ways to combine material properties with different functionalities for various applications. In the electrospinning process, a high voltage is applied between a polymeric solution in a syringe and a metallic collector. When the voltage applied reaches a threshold limit the electrostatic forces overcome surface tension of the solution, the hemispherical surface of the drop in the tip of the syringe (Taylor's cone) elongates and an electrically charged solution jet is generated. As the solution droplet passes from the jet to the collector, the solvent evaporates. Solid fibres are formed and collected in the form of non-woven mats when using a static metallic collector [1].

Electrospun nanofibres have shown great potential for many biomedical applications, including the fabrication of scaffolds for tissue engineering, wound dressing, immobilised enzymes and controlleddelivery of drugs [2,3]. Solution, process and environmental parameters can be tuned to achieve desirable properties of the electrospun fibres [4,5]. Among the parameters of the solution are the concentration, viscosity, conductivity, polymer molecular weight and surface tension. Solvent performs two crucial roles, one is to solvate the polymer molecules ready to form the electrified jet and the other is to carry the solvated polymer molecules towards the collector [1]. Within the process parameters are the flow rate, voltage applied, the diameter of the injector tip, the tip and the tipcollector distance. The voltage applied is linked to the distance between the tip of the needle and the collector surface or the metal plate. The higher the electrical potential, the thinner and lighter are the fibres [5]. The injection distance is measured from the tip of the needle to the collecting platform connected to ground. There is a minimum distance required for the fibres to reach the collector properly but also if the distance is too large the fibres cannot be deposited [3,6]. The collector of fibres or metal plate may be fixed or mobile and can be tuned to achieve the fibre thickness [3]. The most relevant environmental parameters are the temperature and humidity [1]. 
Electrospinning parameters determine the diameter, fibre morphology, the amount of polymer which reaches the collector and the number of defects in the fibres, as well as their structural characteristics [5]. Electrospun fibre mechanical properties such as modulus, strength and elasticity, strongly depend on the initial conditions of the system, thus by proper handling of these parameters, it is possible to produce fibres with the desired characteristics [7].

Furthermore, a variety of works have showed the potential use of polyamide scaffolds due to the ability of the physical properties of these polymers to suit biomedical applications [8-10]. Polyamide 6,6, derived from 1,6-hexamethylene diamine and adipic acid, is a semicrystalline polymer which has good thermal stability and mechanical strength, and it is an important engineering thermoplastic [7,912]. When polyamides are mixed in a protonic solvent, functional groups yield reactions of chemical exchange resulting in a poly-electrolytic behaviour of the solution [13]. This makes polyamide 6,6 a suitable polymer for electrospinning processing.

Because the dependence of the crystalline structure on the mechanical properties of the electrospun fibres [10,11], this research focuses on the crystallinity features provided by the variation of electrospinning parameters including voltage applied, solution concentration, distance from the tip of the needle to the collector surface and flow rate. The parameters accounting for significant changes on the crystallinity of the polyamide 6,6 nanofibres obtained by differential scanning calorimetry (DSC) are presented. Structural changes on the electrospun fibres were also observed by analysing Fourier transform infrared (FTIR) and Raman spectra.

\section{Results and Discussion}

Crystallinity is a property directly associated to the structural changes of polymers. The procedure used to obtain the percentage of crystallinity from DSC is explained in the experimental section. Repeatability of the crystallinity results was corroborated by testing 30 samples under the same working conditions (low solution concentration, $20 \mathrm{kV}$ voltage applied, $15 \mathrm{~cm}$ tip-collector distance, at $5 \mathrm{ml} / \mathrm{h}$ flow rate). This sample space is suitable according to the law of large numbers and the distribution of the crystallinity percentage values are shown in Figure 1. The resulting average crystallinity percentage was 37.7 with a standard deviation of 1.3 . The asymmetry coefficient was -0.3709 , which is almost a symmetrical distribution with a tendency to the left and a kurtosis of -0.9526 , close to normal (see the upper graph in Figure 1). Therefore, the sampling results are close to a normal distribution. This implies that electrospinning process produces independent and reproducible results with less than $3.5 \%$ error margin of crystallinity when working under the same conditions. Crystallinity percentage results obtained by DSC of the samples produced at two different voltages, two flow rate velocities, two tip to collector distances and three solution concentrations are found in Tables 1 and 2. The values of these parameters are included in the experimental section.

Considering only the flow rate variations in Tables 1 and 2, the crystallinity percentage shows increases when the electrospun fibres were obtained at high flow rate when using low solution concentration, $15 \mathrm{kV}$ voltage applied, 15 and $20 \mathrm{~cm}$ of distance. On the other hand, a decrease of crystallinity percentage was attained when using a high flow rate, low solution concentration, $20 \mathrm{kV}$ 
voltage applied, 15 and $20 \mathrm{~cm}$ of distance. Thus flow rate modification did not produce any consistent effect on the nanofibre crystallinity percentage. Taking into account only changes in the tip-collector separation distance, the results did not show any systematic variation of the crystallinity percentage when modifying the fibre collection distance. As an example, Tables 1 and 2 show that crystallinity percentage can be enhanced when operating at $15 \mathrm{kV}$, with a low solution concentration and at a flow rate of $2.5 \mathrm{ml} / \mathrm{h}$ while when operating at $20 \mathrm{kV}$, low solution concentration and at a flow rate of 2.5 $\mathrm{ml} / \mathrm{h}$ this property is reduced.

Figure 1. Crystallinity percentage distribution of the 30 samples obtained under the same electrospinning conditions.

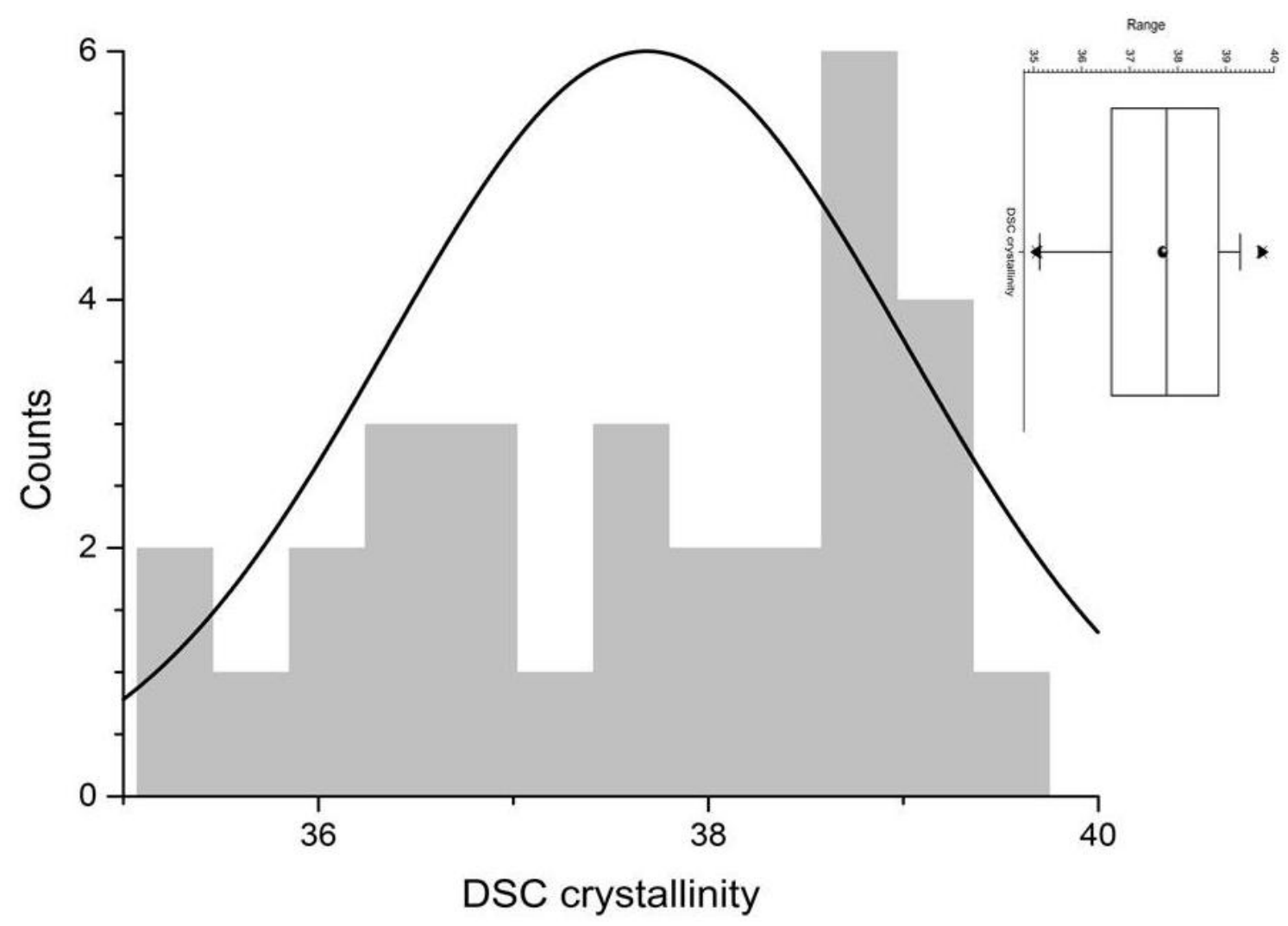

Table 1. DSC results of the fibres obtained at $15 \mathrm{kV}$.

\begin{tabular}{|c|c|c|c|c|c|c|c|c|c|c|c|c|}
\hline \multirow{3}{*}{$\begin{array}{l}\text { Solution Concentration } \\
\text { Distance, } \mathrm{cm} \\
\text { Flow rate, } \mathrm{ml} / \mathrm{h}\end{array}$} & \multicolumn{4}{|c|}{ Low } & \multicolumn{4}{|c|}{ Medium } & \multicolumn{4}{|c|}{ High } \\
\hline & \multicolumn{2}{|c|}{15} & \multicolumn{2}{|c|}{20} & \multicolumn{2}{|c|}{15} & \multicolumn{2}{|c|}{20} & \multicolumn{2}{|c|}{15} & \multicolumn{2}{|c|}{20} \\
\hline & 2.5 & 5.0 & 2.5 & 5.0 & 2.5 & 5.0 & 2.5 & 5.0 & 2.5 & 5.0 & 2.5 & 5.0 \\
\hline Crystallinity \% & 31.5 & 38.9 & 36.0 & 37.2 & 41.4 & 40.7 & 35.3 & 38.8 & 36.7 & 36.1 & 40.0 & 40.3 \\
\hline
\end{tabular}

Table 2. DSC results of the fibres obtained at $20 \mathrm{kV}$.

\begin{tabular}{|c|c|c|c|c|c|c|c|c|c|c|c|c|}
\hline Solution Concentration & \multicolumn{4}{|c|}{ Low } & \multicolumn{4}{|c|}{ Medium } & \multicolumn{4}{|c|}{ High } \\
\hline Distance, $\mathrm{cm}$ & \multicolumn{2}{|c|}{15} & \multicolumn{2}{|c|}{20} & \multicolumn{2}{|c|}{15} & \multicolumn{2}{|c|}{20} & \multicolumn{2}{|c|}{15} & \multicolumn{2}{|c|}{20} \\
\hline Flow rate, $\mathrm{ml} / \mathrm{h}$ & 2.5 & 5.0 & 2.5 & 5.0 & 2.5 & 5.0 & 2.5 & 5.0 & 2.5 & 5.0 & 2.5 & 5.0 \\
\hline Crystallinity $\%$ & 44.1 & 41.0 & 40.7 & 38.2 & 42.8 & 44.4 & 41.1 & 39.4 & 36.9 & 43.6 & 38.6 & 39.7 \\
\hline
\end{tabular}


Electric field voltage variations have a considerable effect on the crystallinity percentage of the polyamide 6,6 fibres. At high voltage applied the crystallinity obtained by DSC resulted in greater values than when operating at low voltage and the rest of the parameters were kept constant. For example, in the fibres obtained using a medium concentration solution, flow rate of $2.5 \mathrm{ml} / \mathrm{h}$ and $15 \mathrm{~cm}$ of tip-collector separation, the crystallinity percentage changes from $41.40 \pm 0.014$ to $42.83 \pm 0.015$ when the electric field voltage varies from $15 \mathrm{kV}$ to $20 \mathrm{kV}$. This behaviour is consistent in most experiments except when using a high solution concentration, $20 \mathrm{~cm}$ distance of collection and the two different flow rates. The close values of the samples obtained at these two different voltages applied indicate the limit conditions that favour crystallisation of polymer.

The variation of solution concentration did not provide a regular effect on the crystallinity of the fibres. However, when keeping the flow rate at $2.5 \mathrm{ml} / \mathrm{h}, 15 \mathrm{~cm}$ of separation and voltage of $20 \mathrm{kV}$, crystallinity percentage changed from $44.10 \pm 0.015$ to $36.89 \pm 0.013$ when the solution concentration was varied from low to high. The dramatic effect on the crystallinity can be explained in the following manner. When using a low concentration solution the polymer chains have more mobility resulting in a higher molecular orientation during electrospinning process as compared to fibres obtained from highly concentrated solutions [13]. This behaviour is not noticeable at larger tip to collector distances because increasing the jet trajectory favoured the orientation of polymer chains and crystallinity.

Crystallinity percentage data of the electrospun samples were processed in Minitab software to obtain the main effects plots, included in Figure 2. The values displayed in this figure represent the mean of each parameter. The design of experiment consists of three factors (voltage, distance, flow) with two levels and one factor (solution concentration) with three levels. From these plots we can determine that the factors which have a considerable influence on the crystallinity of the samples are those with the highest slopes; therefore, both voltage and concentration provided the major effects on the tested response when compared to the rest of the parameters.

Figure 2. Main effects of the electrospinning parameters.

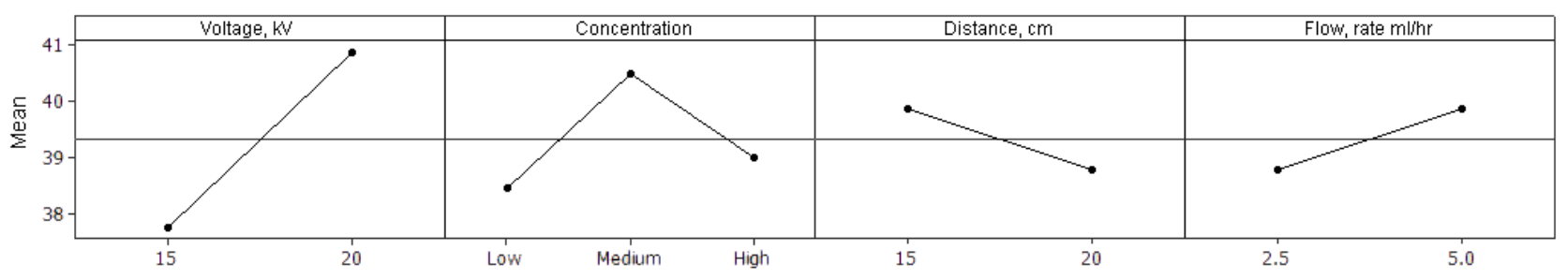

SEM allowed the determination of the polyamide 6,6 nanofibre diameters produced by the variations of the electrospinning parameters. Figure 3 shows the entanglement and disorientation of the fibres due to the electrospinning set up employed in these experiments. The fibre diameters were measured from these SEM images using the image analysis software Image ${ }^{\circledR}$. The fibre diameter of the samples which resulted in the highest, medium and lowest crystallinity percentage are reported in Table 3. The three samples analysed resulted in similar fibre diameters due to the combination of the parameters employed for the fibre electrospinning. 
Figure 3. SEM images of the electrospun fibres with the (a) highest, (b) medium and (c) lowest crystallinity percentage.

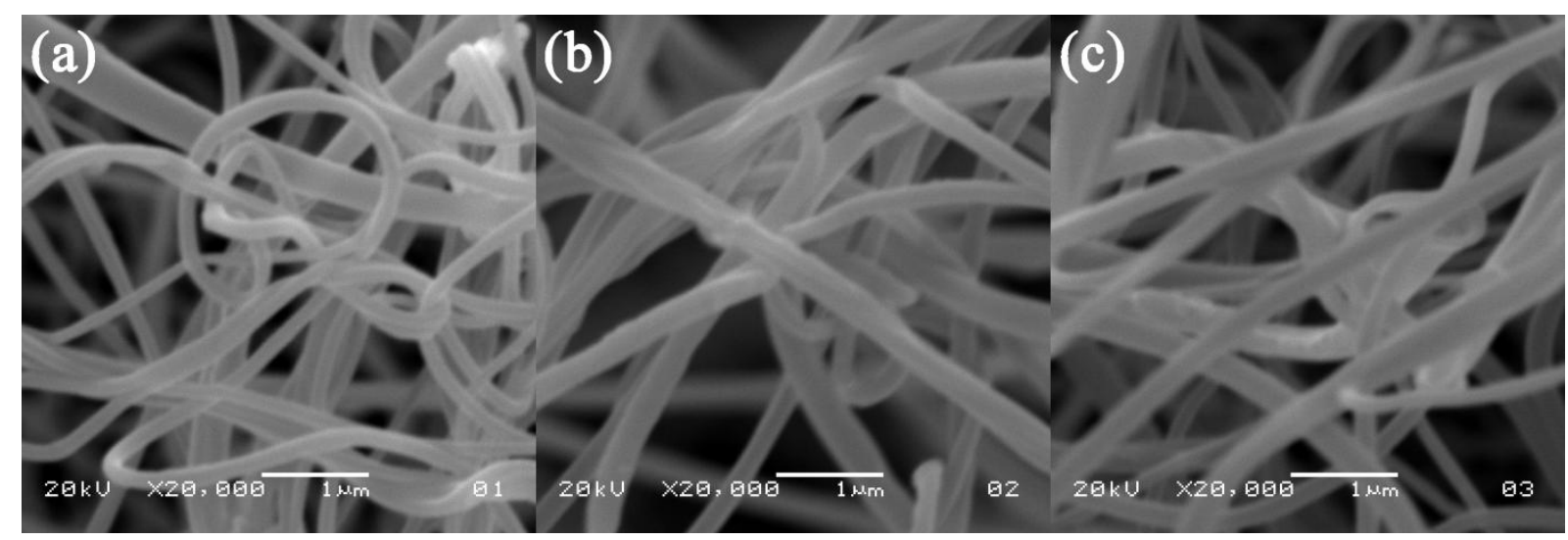

Table 3. Diameters obtained from the polyamide electrospun fibres.

\begin{tabular}{|cc|}
\hline Crystallinity percentage & Average diameter $(\mathrm{nm})$ \\
\hline $31.52 \pm 0.011$ & $237 \pm 30$ \\
$39.36 \pm 0.014$ & $226 \pm 25$ \\
$44.37 \pm 0.016$ & $285 \pm 94$ \\
\hline
\end{tabular}

The characteristic functional groups of polyamide 6,6 were analysed by FTIR spectroscopy [14,15] and they are summarised in Table 4. Figure 4 shows three of the normalised FTIR spectra obtained using different solution concentration while the rest of the working conditions remained constant. The FTIR spectra show similar features regarding the polar group vibrations of polyamide 6,6 . However, there are intensity modifications in the peaks related to carbon skeleton vibrations as depicted in the highlighted area of Figure 4a. Modifications in the peaks located at 1020 and $1095 \mathrm{~cm}^{-1}$ corresponding to the $\mathrm{C}-\mathrm{C}$ stretch are clearly seen in Figure $4 \mathrm{~b}$. This finding may be correlated with changes in the crystal structure. Vasanthan et al. found that librational motion of methylene segments can be associated to changes in the packing of the crystalline polymer chains [15].

Functional groups of polyamide 6,6 identified in the Raman spectrograms $[17,18]$ are included in Table 4. Figure 5 shows the normalised Raman spectra of the fibres obtained at different solution concentration and the rest of the working conditions were kept constant. This figure shows that the peaks in the 1150-1600 $\mathrm{cm}^{-1}$ region are overlapped and as the solution concentration is increased the peaks are better defined and their intensities are also increased. Furthermore, the peak located at $\sim 1386$ $\mathrm{cm}^{-1}$ is redshifted. This change to lower wavenumber was also dependent of solution concentration. Some peak intensities were also increased as the solution is more concentrated (1060, 1130 and 1297 $\mathrm{cm}^{-1}$ ). Modifications of Raman peaks in the range of $800-1600 \mathrm{~cm}^{-1}$ mainly related to the carbon backbone have been previously associated with crystalline structural changes of polyamides [18-20]. Methylene stretches in the $2800-2900 \mathrm{~cm}^{-1}$ region corresponding to methylene stretches have been also related in terms of crystal morphology [18]. 
Figure 4. FTIR spectra of polyamide fibres obtained at $15 \mathrm{kV}$, distance of $15 \mathrm{~cm}$ and at 2.5 $\mathrm{ml} / \mathrm{hr}$ flow rate.
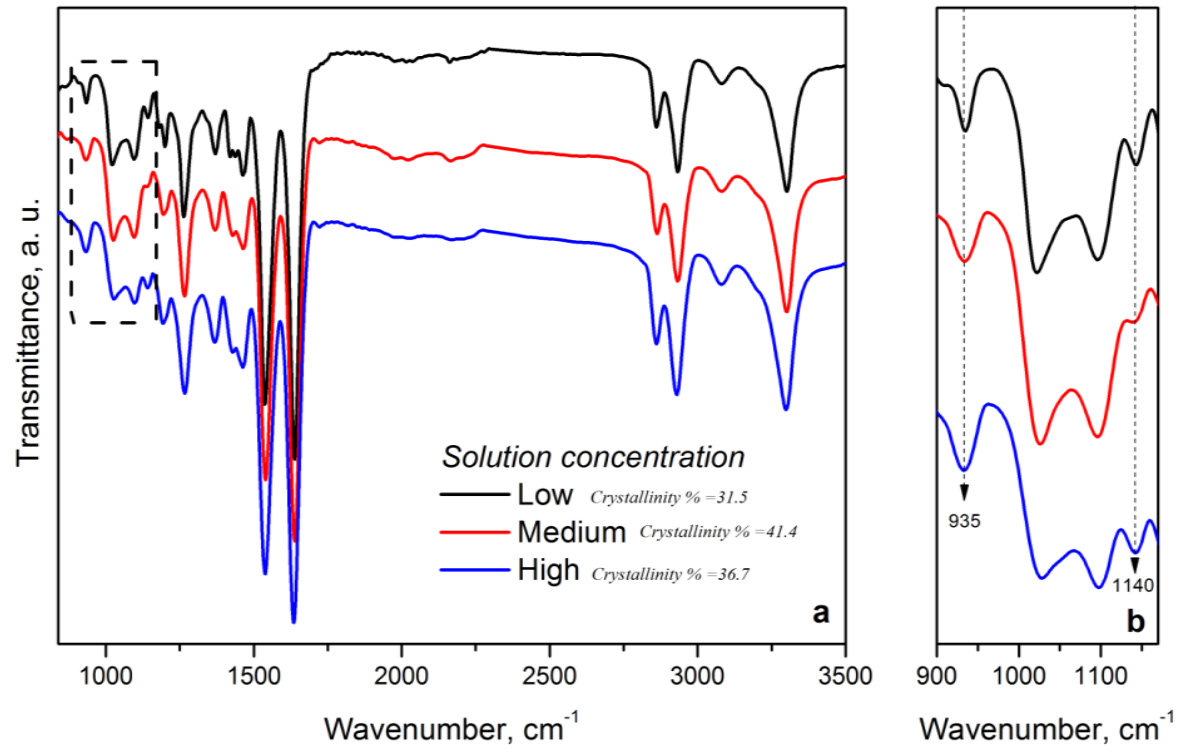

Table 4. Main peaks observed in the electrospun fibres.

\begin{tabular}{|ccc|}
\hline IR Position, $\mathrm{cm}^{-1}$ & Raman Position, $\mathrm{cm}^{-1}$ & Peak Assignment \\
\hline 937 & 950 & $\mathrm{C}-\mathrm{C}=\mathrm{O}$ stretch \\
$1020-1150$ & $1050-1130$ & C-C stretch \\
1200 & 1205 & C-N-H wagging (amide III) \\
- & 1235 & $\mathrm{~N}-\mathrm{H}$ wagging \\
1365 & - & $\mathrm{C}-\mathrm{N}$ stretch \\
$1400-1500$ & $1270-1470$ & $\mathrm{CH}$ deformations \\
1535 & & $\mathrm{C}=\mathrm{O}-\mathrm{N}-\mathrm{H}$ (amide II) \\
1635 & 1638 & $\mathrm{C}=\mathrm{O}$ (amide I) \\
2860 & 2865 & $\mathrm{CH}$ stretch \\
2930 & 2915 & $\mathrm{CH}$ stretch \\
$3025-3400$ & - & $\mathrm{N}-\mathrm{H}$ stretch \\
\hline
\end{tabular}

Furthermore, FTIR peaks related to vibrational modes of functional groups in the crystalline and amorphous domains of polyamide 6,6 are located at $\sim 935$ and $\sim 1140 \mathrm{~cm}^{-1}$, respectively [16]. As seen in Figure $6 \mathrm{a}$ and $6 \mathrm{~b}$, the crystalline peak broadness and intensity increases as the solution concentration is increased (notice that the colours follow the same fashion as the samples labelled in the figures above). However, the intensity of the amorphous peak has a different behaviour, where the fibres with the highest crystallinity percentage in that set of samples exhibited the weakest peak. In addition, the crystalline band at $935 \mathrm{~cm}^{-1}$ can be associated the with the band located at $950 \mathrm{~cm}^{-1}$ in Raman spectra $[17,10]$. Figure $6 \mathrm{c}$ displays that the intensity of these peaks increase as the solution concentration is higher. Zimba et al. correlated the amorphous FTIR peaks found at 1140, 1232 and $1338 \mathrm{~cm}^{-1}$ with the peaks present in Raman at similar frequencies. Our spectra only show the peak at $\sim 1230 \mathrm{~cm}^{-1}$ (see Figure 5d) [21]. Table 5 shows comparative measurements between the FTIR and Raman peak heights 
and the ratio of crystalline to amorphous heights. The evolution of these crystallinity ratios is in agreement with the results obtained from DSC, indicating that the solution concentration has an effect on the structural changes of crystalline domains.

Table 5. Crystalline and amorphous peak heights of vibrational spectroscopies.

\begin{tabular}{|c|ccc|ccc|}
\hline \multirow{2}{*}{ Solution Concentration } & \multicolumn{3}{|c|}{ FTIR } & \multicolumn{3}{c|}{ Raman } \\
\cline { 2 - 7 } & $H_{935}$ & $H_{1140}$ & $H_{935 / 1140}$ & $H_{955}$ & $H_{1230}$ & $H_{955 / 1230}$ \\
\hline Low & 0.057 & 0.044 & 1.315 & 0.037 & 0.033 & 1.133 \\
Medium & 0.054 & 0.017 & 3.251 & 0.042 & 0.032 & 1.322 \\
High & 0.071 & 0.032 & 2.228 & 0.065 & 0.053 & 1.226 \\
\hline
\end{tabular}

Figure 5. Raman spectra of polyamide fibres obtained at $15 \mathrm{kV}$, distance of $15 \mathrm{~cm}$ and at $2.5 \mathrm{ml} / \mathrm{hr}$ flow rate.

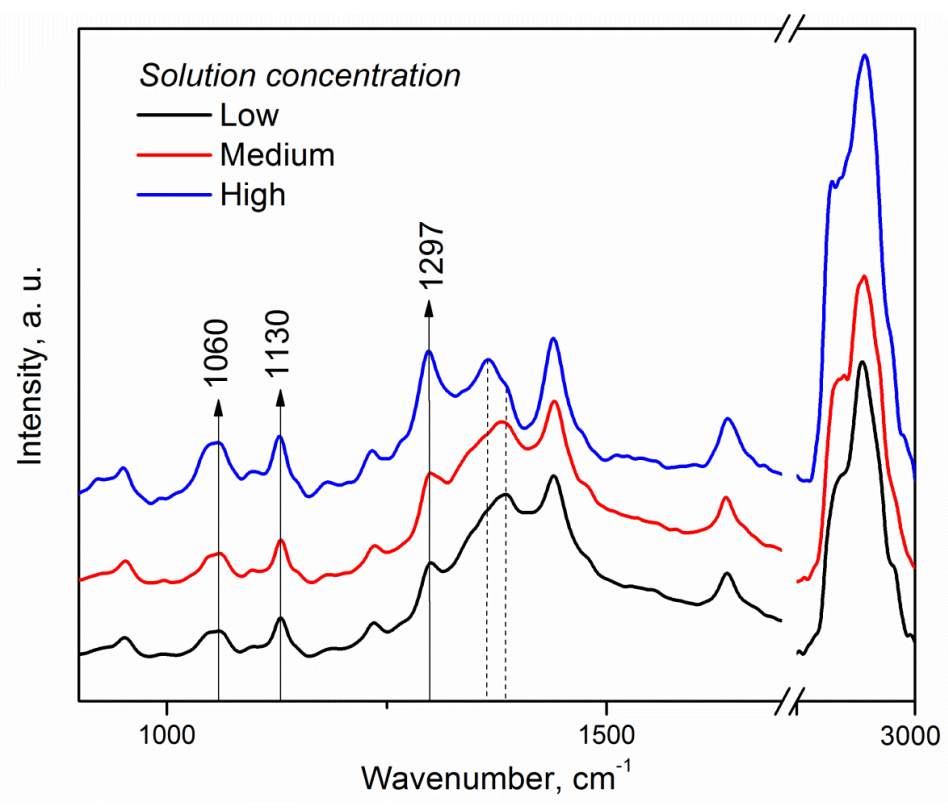

Figure 6. FTIR and Raman peaks of crystalline and amorphous contributions.
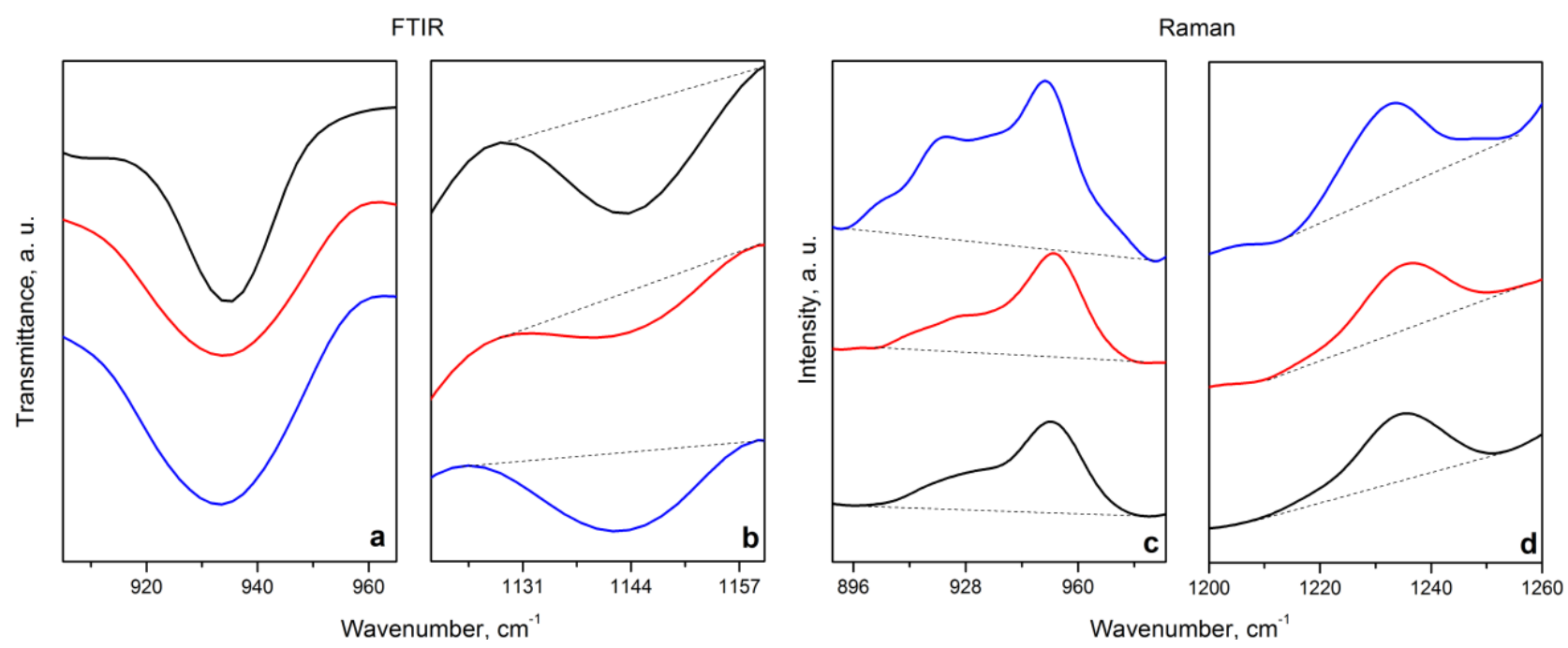


\section{Experimental Section}

\subsection{Electrospinning of Nylon 6,6}

Three polymer solutions were prepared by dissolving polyamide 6,6 pellets (Ultramid ${ }^{\circledR} \mathrm{A} 3 \mathrm{~K}$, BASF) in formic acid (88\%, Sigma-Aldrich) and kept under stirring for four hours at $60{ }^{\circ} \mathrm{C}$. Then, the solution was cooled to room temperature for being deposited into a $5 \mathrm{ml}$ syringe (21 gauge, 1" needle). The flow rate of the solution was controlled using a syringe pump (KDScientific 101) at two dose velocities. Two different voltages were applied directly to the needle and two tip-collector distances were employed. A copper plate wrapped with aluminium foil was used. Table 6 shows the electrospinning parameters of the experiments.

Table 6. Parameter values of the electrospinning process to produce polyamide 6,6 fibres.

\begin{tabular}{|c|c|c|c|}
\hline Parameter & \multicolumn{3}{|c|}{ Working condition } \\
\hline $\begin{array}{c}\text { Solute concentration, } g \\
\text { (diluted in } 20 \mathrm{~g} \text { of formic acid) }\end{array}$ & $\begin{array}{l}4.0 \\
\text { (low) }\end{array}$ & $\begin{array}{c}4.5 \\
\text { (medium) }\end{array}$ & $\begin{array}{l}5.0 \\
\text { (high) }\end{array}$ \\
\hline Electric field voltage, $\mathrm{kV}$ & 15 & & 20 \\
\hline Tip-collector distance, $\mathrm{cm}$ & 15 & & 20 \\
\hline Flow rate, $\mathrm{ml} / \mathrm{h}$ & 2.5 & & 5.0 \\
\hline
\end{tabular}

\subsection{Polyamide 6,6 characterisation techniques}

DSC tests were carried out in a TA Instruments DSC Q2000. The samples were tested from 25 to $280^{\circ} \mathrm{C}$ at a heating rate of $10^{\circ} \mathrm{C} / \mathrm{min}$. A 5 minute isotherm was applied for subsequently cooling down the sample to $25^{\circ} \mathrm{C}$, using nitrogen as the purge gas. Polyamide 6,6 crystallinity determination in this experiment was performed by integration of the melting curves in the region from $200^{\circ} \mathrm{C}$ to $274^{\circ} \mathrm{C}$ using OriginPro ${ }^{\circledR}$. The crystallinity percentage is given by:

$$
X_{c}=\frac{\Delta H_{m}}{\Delta H_{m}^{0}} \times 100 \%
$$

where $\Delta H_{m}$ and $\Delta H^{0} m(197 \mathrm{~J} / \mathrm{g})$ are the enthalpies of the nanocomposite and purely crystalline polyamide 6,6 respectively $[11,12]$.

The morphology of the fibres was observed by SEM using a JSM-6060LV JEOL microscope at an accelerating voltage of $20 \mathrm{kV}$. The samples were mounted on cupper stubs and were vacuum-coated with gold at $7 \times 10^{-2}$ mbar using Argon in a sputter coater EMS 550. SEM images allowed the determination of the average diameter of 30 polyamide 6,6 fibres obtained using the software Image ${ }^{\circledR}$. 
The characteristic groups of polyamide 6,6 were analysed using a FTIR Bruker Vector 33 spectrometer in attenuated total reflectance mode, with a spectral resolution of $1 \mathrm{~cm}^{-1}$. Raman spectroscopy was performed in a Raman Senterra Bruker machine with a $100 \times$ microscope objective, a laser emitting at $785 \mathrm{~nm}$ and a resolution $1 \mathrm{~cm}^{-1}$.

\section{Conclusions}

Structural properties of electrospun fibres can be modified through variation of the characteristic electrospinning parameters. The electrospun fibres obtained in this work produced repeatable results on the crystallinity percentage with a deviation of $3.5 \%$. This property was significantly affected by the voltage applied. Modifications of the flow rate, the tip-collector distance and solution concentration did not have consistent effects on the electrospun fibres fabricated. However, FTIR and Raman spectroscopies aided the identification of structural crystalline changes on the polyamide 6,6 fibres when varying the solution concentration used for the electrospinning process. In addition, the evolution of the crystalline and amorphous peaks in these spectroscopies are in aggrement. Understanding of the effects provided on the crystalline features of polyamide fibres is useful for tuning scaffold mechanical properties.

\section{Acknowledgments}

The authors appreciate the support of F. Fernandez for his help in the electrospinning instrumental adjustment, G. Hernández Padrón for her assistance in IR and Raman spectroscopies and A. del Real López for her assistance in SEM, at Centro de Física Aplicada y Tecnología Avanzada.

\section{Conflicts of Interest}

The authors declare no conflict of interest.

\section{References and Notes}

1. Huang M.-Z.; Zhang Z.-Y.; Kotaki M.; Ramakrishna S. A review on polymer nanofibers by electrospinning and their applications in nanocomposites. Composites Science and Technology 2003, 63(15), 2223-2253.

2. Liang, D.; Hsiao, B.S.; Chu, B. Functional electrospun nanofibrous scaffolds for biomedical applications. Advanced Drug Delivery Reviews 2007, 59(14), 1392-1412.

3. Kumbar S.G., Nukavarapu S.P., James R., Hogan M.V., Laurencin C.T. Recent patents on electrospun biomedical nanostructures: an overview. Recent Patents on Biomedical Engineering 2008, $1,68-78$

4. Bellan, L.M.; Craighead, H.G. Applications of controlled electrospinning systems. Polymer Advanced Technologies 2011, 22, 304-309.

5. Bhardwaj, N.; Kundu, S.C. Electrospinning: A fascinating fiber fabrication technique, Biotechnology Advances 2010 , 28, 321-347. 
6. Yördem, O.S.; Papila, M.; Menceloğlu, Y.Z. Effects of electrospinning parameters on polyacrylonitrilenanofiber diameter: an investigation by response surface methodology, Materials \& Design 2008, 29, 34-44.

7. Baji, A.; Mai, Y.-W.; Wong, S.-C.; Abtahi, M.; Chen, P. Electrospinning of polymer nanofibers: Effects on oriented morphology, structures and tensile properties. Composites Science and Technology 2010, 70(5), 703-718.

8. Simpson, D.G.; Jha, B.S.; Ayres, C.E.; Bowman, J.R.; Telemeco, T.A.; Sell, S.A.; Bowlin, G.L. Electrospun collagen: A tissue engineering scaffold with unique functional properties in a wide variety of applications. Journal of Nanomaterials 2011, 348268, 15 pp.

9. Nuhiji, E.; Wong, C.S.; Sutti, A.; Lin, T.; Kirkland, M.; Wang, X. Biofunctionalization of 3D nylon 6,6 scaffolds using a two-step surface modification. ACS Applied Materials and Interfaces 2012, 4(6), 2912-2919.

10. Navarro-Pardo, F.; Martínez-Barrera, G.; Martínez-Hernández, A.L.; Castaño, V.M.; RiveraArmenta, J.L.; Medellín-Rodríguez, F.; Velasco-Santos, C. Nylon 6,6 electrospun fibres reinforced by amino functionalised $1 \mathrm{D}$ and 2D carbon. IOP conference series: Materials Science and Engineering 2012, 40, 012023.

11. Navarro-Pardo, F.; Martínez-Barrera, G.; Martínez-Hernández, A.L.; Castaño, V.M.; RiveraArmenta, J.L.; Medellín-Rodríguez, F.; Velasco-Santos, C. Effects on the thermo-mechanical and crystallinity properties of nylon 6,6 electrospun fibres reinforced with one dimensional (1D) and two dimensional (2D) carbon. Materials 2013, 6(8), 3494-513.

12. Nirmala, R.; Navamathavan, R.; Kang, H.-.S.; El-Newehy, M.H.; Kim, H.Y. Preparation of polyamide-6/chitosan composite nanofibers by a single solvent system via electrospinning for biomedical applications. Colloids and Surfaces B: Biointerfaces 2011, 83(1), 173-178.

13. Ero-Phillips, O.; Jenkins, M.; Stamboulis, A. Tailoring crystallinity of electrospun PLLA fibres by control of electrospinning parameters. Polymers 2012, 4(3), 1331-1348.

14. Guerrini, L.M.; Branciforti, M.C.; Canova, T.; Bretas, R.E.S. Electrospinning and characterization of polyamide 66 nanofibers with different molecular weights, Materials Research 2009, 12(2), 181-190.

15. Vasanthan, N.; Murthy, N.S.; Bray, R.G. Investigation of brill transition in nylon 6 and nylon 6,6 by infrared spectroscopy. Macromolecules, 1998, 31(23), 8433-8435.

16. Gonçalves, E.S.; Poulsen, L.; Ogilby, P.R. Mechanism of the temperature-dependent degradation of polyamide 66 films exposed to water. Polymer Degradation and Stability, 2007, 92(11), 1977 1985.

17. Cho L.L. Identification of textile fiber by Raman microspectroscopy, Forensic Science Journal 2007, 6,55-62

18. Menchaca C.; Manoun B.; Martinez-Barrera G.; Castaño V.M.; Lopez-Valdivia H. In situ hightemperature Raman study of crystalline Nylon 6,12 fibers gamma-irradiated in argon atmosphere, Journal of Physics and Chemistry of Solids 2006, 67,2111-2118.

19. Colomban, P.; Herrera Ramirez, J.M., Paquin, R., Marcellan, A., Bunsell, A. Micro-Raman study of the fatigue and fracture behaviour of single PA66 fibres. Comparison with single PET and PP fibres, Engineering Fracture Mechanics 2006, 7, 2463-2475. 
20. Ferreiro, V.; Depecker, C.; Laureyns, J.; Coulon, G. Structures and morphologies of cast and plastically strained polyamide 6 films as evidenced by confocal Raman microspectroscopy and atomic force microscopy. Polymer 2004, 45(17), 6013-6026.

21. Zimba, C.G.; Rabolt, J.F.; English, A.D. Raman spectroscopic characterization and molecular force field development of a synthetic polyamide: Nylon 66. Macromolecules 1989, 22(6), 2863 2867.

(C) 2014 by the authors; licensee MDPI, Basel, Switzerland. This article is an open access article distributed under the terms and conditions of the Creative Commons Attribution license (http://creativecommons.org/licenses/by/3.0/). 